\title{
Práticas de Administração Pública no ambiente cultural dos países de língua portuguesa
}

por Joaquim Fontes Filho e Roberto da Costa Pimenta

RESUMO: Este artigo tem como objetivo refletir sobre uma agenda de pesquisa com vista a avaliar a adequação das novas práticas e modelos de Administração Pública ao contexto dos países da Comunidade dos Países de Língua Portuguesa (CPLP). Estudos recentes apontam uma tendência de deslocamento do paradigma da New Public Management (NPM), baseada na eficiência, nas técnicas empresariais e no controle de resultados, para a lógica da New Public Governance (NPG), centrada nos mecanismos de coprodução, na criação de redes e processos não hierárquicos, nos acordos tácitos com os diversos atores da sociedade e, notadamente, no princípio de valor público. Nesse artigo, sugerimos uma agenda de investigação com foco na análise dos elementos que condicionam a aplicação da NPG, no contexto da CPLP, e das possíveis barreiras a sua efetiva implantação. Finalizamos com uma discussão acerca da perspectiva de superação dessas barreiras.

Palavras-chave: Administração Pública; Cultura; Modelos de Gestão; Governança Pública

\section{Prácticas de la Administración Pública en el ámbito cultural de los países de habla portuguesa}

RESUMEN: Este artículo tiene como objetivo reflexionar sobre un programa de investigación con el fin de evaluar la adecuación de las nuevas prácticas y modelos de la Administración Pública en el contexto de los países de la Comunidad de lengua portuguesa (CPLP). Estudios recientes apuntan una tendencia al cambio de paradigma de New Public Management (NGP), basado en la eficiencia, en las técnicas empresariales y en el control de resultados, para la lógica de New Public Governance (NPG), centrándose en los mecanismos de coproducción, en la creación de redes y procesos no jerárquicos, acuerdos tácitos con los diversos actores de la sociedad y, en particular, en el principio de valor público. En este artículo, sugerimos un programa de investigación enfocado en el análisis de los elementos que influyen en la aplicación de la NPG, en el contexto de la CPLP, y de las posibles barreras para su efectiva implantación. Finalizamos con una discusión acerca de la perspectiva de superación de estas barreras.

Palabras clave: Administración Pública; Cultura; Modelos de Gestión; Gobernanza Pública 


\section{Practices of public administration in the cultural context of Portuguese speaking countries}

ABSTRACT: This article aims to reflect on a research agenda with a view to assessing the adequacy of the new models and practices of public administration models to the context of the Community of Portuguese speaking countries (in Portuguese, CPLP). Recent studies indicate a depletion trend of the New Public Management (NPM) paradigm, based on efficiency, business techniques and focus on control results, and the emergence of a logic based on public governance, focusing on co-production mechanisms, the creation of networks and non-hierarchical processes, tacit agreements with the various actors in society and, in particular, the principle of public value. In this article, we suggest a research agenda focused on the analysis of the elements that influence the application of the NPG in the context of the CPLP and the possible barriers to their effective implementation, also advancing the discussion about the prospect of overcoming these barriers.

Key words: Public Administration; Culture; Management Models; Public Governance

Joaquim Ruben Fontes Filho

joaquim.rubens@fgv.br

Doutorado em Administração, Fundação Getulio

Vargas/Escola Brasileira de Administração Pública e de Empresas (FGV/EBAPE). Coordenador do Mestrado Profissional em Gestão Empresarial e Professor Adjunto, FGV/EBAPE, CEP 22250-900 - Rio de Janeiro-RJ, Brasil. Doctorado en Administración de Empresas, Fundación Getulio Vargas / Escuela Brasileña de Administración Pública y de Empresas (FGV / EBAPE). Coordinador Profesional del Master en Administración de Empresas y Profesor Adjunto de la FGV / EBAPE, CEP 22250-900 - Rio de Janeiro-RJ, Brasil.

$\mathrm{PhD}$ in Administration, Getulio Vargas Foundation/Brazilian School of Public and Business Administration. Head of the Executive Master's in Business Administration and

Assistant Professor, Getulio Vargas Foundation/Brazilian School of Public and Business Administration, CEP 22250-900 - Rio de Janeiro-RJ, Brazil.

\section{Roberto da Costa Pimenta}

roberto.pimenta@fgv.br

Doutorado em Administração, Fundação Getulio

Vargas/Escola Brasileira de Administração Pública e de Empresas (FGV/EBAPE). Coordenador do Mestrado

Profissional em Gestão Empresarial e Professor Adjunto, FGV/EBAPE, Praia de Botafogo, 190 - 5. ${ }^{\circ}$ andar, sala 508, CEP 22250-900 - Rio de Janeiro-RJ, Brasil.

Doctorado en Administración de Empresas, Fundación Getulio Vargas / Escuela Brasileña de Administración Pública y de Empresas (FGV / EBAPE). Coordinador Profesional del Master en Administración de Empresas y Profesor Adjunto de la FGV / EBAPE, Praia de Botafogo, 190 - $5^{\circ}$ piso, sala 508, CEP 22250-900 - Rio de Janeiro-RJ, Brasil.

$\mathrm{PhD}$ in Administration, Getulio Vargas Foundation/Brazilian School of Public and Business Administration. Head of the Executive Master's in Business Administration and Assistant Professor, Getulio Vargas Foundation/Brazilian School of Public and Business Administration, CEP 22250-900 - Rio de Janeiro-RJ, Brazil. 
Este texto recupera e compartilha uma reflexão há muito estabelecida por diversos pensadores da administração sobre a possibilidade de importação de modelos e práticas administrativas entre países e culturas. Mas a proposta aqui é um pouco mais focalizada, pretendendo trazer à discussão um momento específico das inovações no conhecimento do setor público desenvolvido e implantado nos países anglo-saxônicos e sua utilidade para a Comunidade dos Países de Língua Portuguesa (CPLP), tendo como base um cotejamento entre aspectos institucionais e culturais.

As reformas administrativas implementadas no período recente nos países que integram a CPLP podem ser entendidas como uma resposta aos desafios impostos pela interdependência e complexidade do ambiente global. A modernização da Administração Pública, das empresas e das organizações não governamentais tem se estabelecido como reflexo da necessidade de inserção dos países da Comunidade em um novo contexto de compartilhamento mundial.

A expectativa dos países menos avançados em capacidade de gestão se inspira na possibilidade de apropriação desse conhecimento específico a partir de experiências levadas a efeito em organizações dos países desenvolvidos, de modo a assegurar ganhos com a prática e o saber já aplicado a outros contextos.

Há riscos e limitações associados a essa perspectiva de transposição do conhecimento administrativo. O principal deles diz respeito a propagação genérica e acrítica de uma lógica administrativa baseada numa racionalidade dominante, que, na maioria das vezes, desconsidera os elementos históricos e institucionais, bem como a diversidade cultural de ambientes distintos daquele ao qual o modelo se origina.

Nesse artigo, sugerimos uma agenda de investigação com foco na análise dos elementos que condicionam a aplicação da moderna gestão pública no contexto da CPLP e das possíveis barreiras a sua efetiva implantação.

\section{Paradigmas da Administração Pública - situando as mudanças}

Nas últimas três décadas, o campo das teorias e paradigmas da Administração Pública esteve marcado pelo apogeu da visão gerencialista e tecnocrática trazida pela New Public Management (NPM) ou Nova Gestão Pública. Para muitos autores, contudo, a NPM mostra-se cada vez mais anacrônica e incapaz de responder às novas características do Estado moderno, inserido em um mundo marcado pela pluralidade das demandas da sociedade e esgarçamento, em muitas dimensões, das fronteiras nacionais.

Uma análise da produção acadêmica do período mostra o crescimento do interesse pela NPM e, mais recentemente, pela New Public Governance (NPG), a Nova Governança Pública (ver Gráfico I). Indica também que, apesar das críticas, a NPM evidencia ainda um grande interesse de pesquisa, provavelmente relacionado a sua difusão.
Há riscos e limitações associados a essa perspectiva de transposição do conhecimento administrativo. O principal deles diz respeito a propagação genérica e acrítica de uma lógica administrativa baseada numa racionalidade dominante. 
Gráfico I Produção acadêmica sobre a New Public Management e Public Governance (antes de 1990 a 2015)

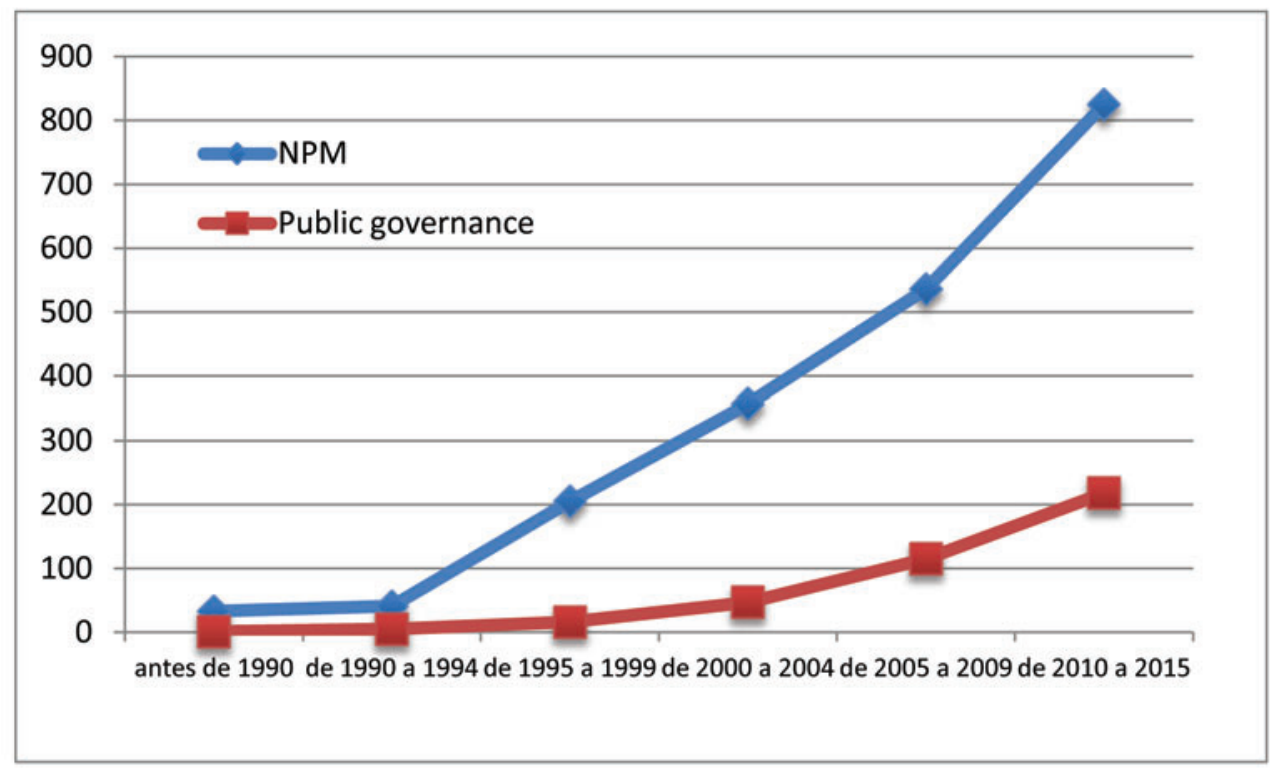

Fonte: Análise publicações sistema EBSCO (não cumulativo).

O estudo sistemático da Administração Pública é relativamente recente, acumulando, segundo Lynn Jr. (2005), cerca de 50 anos, para quem o marco pode ser situado na inclusão nos currículos nas escolas de políticas públicas americanas e nas reformas promovidas na Grã-Bretanha e Nova Zelândia.

Motta (2013), por sua vez, remonta ao Século XVIII, na Prússia, o início de modelos organizados de padronização e transmissão de conhecimentos na área, então focados em aspectos de controle, finanças públicas e na comunicação das ordens públicas, reconhecendo, contudo, que a natureza rudimentar dos conhecimentos eram ainda pouco úteis na solução dos problemas.

A preocupação com uma estrutura de conhecimento para a Administração Pública pode, também, ser localizada nas propostas de Woodrow Wilson, em 1887, posteriormente eleito presidente americano, que defendeu a independência e profissionalização da Administração Pública ante o sistema político, dicotomia ainda hoje objeto de debates, e a utilização de práticas privadas de gestão (Motta, 2013), antecipando em mais de 100 anos os movimentos gerencialistas. Quarenta anos depois a dificuldade de alguma forma permanecia, quando os preceitos de Weber de formalização da burocracia ganharam voz.

No Brasil, Keinert analisou a evolução das ideias e práticas que basearam a Administração Pública no país até 1994, propondo que essa evolução ocorreu segundo uma sequência de paradigmas (ver Quadro I).

Interessante observar que o mapa traçado pela autora termina exatamente no ano que pode ser classificado como o início da difusão das 
práticas da NPM no Brasil. Em 1989, surgiu o denominado Consenso de Washington, um conjunto de dez propostas de natureza liberal - a exemplo das privatizações, liberalização do comércio e do investimento externo, desregulamentação, e política fiscal -, coordenadas pelo Banco Mundial, Fundo Monetário Internacional e Departamento do Tesouro americano, para os países do continente americano envolvidos com problemas de endividamento externo. No ano seguinte, com a eleição no Brasil do presidente Fernando Collor de Mello, a implementação de políticas públicas baseadas nas propostas do referido Consenso criaram as bases para as reformas estruturais que se iniciariam cinco anos depois com o Plano de Reforma do Aparelho de Estado.

A NPM representa um conjunto de premissas e valores sobre como organizações públicas devem ser constituídas, organizadas, gerenciadas e, semelhante aos negócios privados, como devem funcionar (Diefenbach, 2009). Ao focar na eficiência, no controle e na gestão por resultados, a NPM adota uma perspectiva tecnocrática, tendo como base uma independência necessária entre a política e a gestão pública, revisitando assim a dicotomia política-Administração Pública.

Para Ferlie et al. (2005), a NPM remete a um conjunto complexo e variado de transformações focadas no controle de custos, eficiência, na capacidade orçamentária e descentralização do poder gerencial, além da criação de mecanismos de mercado baseados em novas formas contratuais

Quadro 1 Principais paradigmas que configuraram a Administração Pública no Brasil

\begin{tabular}{|c|l|l|l|}
\hline Paradigma & Período & Contexto institucional & $\begin{array}{l}\text { Características do campo da } \\
\text { Administração Pública }\end{array}$ \\
\hline \multirow{2}{*}{ Ciência Jurídica } & $1900-1929$ & Estado regulador-liberal & $\begin{array}{l}\text { Administração Pública como } \\
\text { ciência jurídica }\end{array}$ \\
\hline $\begin{array}{c}\text { Ciência } \\
\text { Administrativa }\end{array}$ & $1946-1964$ & $\begin{array}{l}\text { Administração para o } \\
\text { desenvolvimento }\end{array}$ & Resenvolvimentismo \\
\cline { 2 - 4 } & $1965-1979$ & Estado intervencionista & $\begin{array}{l}\text { Racionalidade e competência } \\
\text { técnicas }\end{array}$ \\
\hline \multirow{2}{*}{$\begin{array}{c}\text { Ciência Política } \\
\text { Estado administrativo }\end{array}$} & $1980-1989$ & Mobilização social & $\begin{array}{l}\text { Democratização } \\
\text { Conflito de interesses } \\
\text { Recursos escassos }\end{array}$ \\
\hline $\begin{array}{c}\text { Administração } \\
\text { Pública }\end{array}$ & $1990-\ldots$ & $\begin{array}{l}\text { Redefinição do papel do } \\
\text { Estado }\end{array}$ & $\begin{array}{l}\text { Capacidade política aliada à } \\
\text { competência técnica }\end{array}$ \\
\hline
\end{tabular}

Fonte: Keinert, 1994 
e mecanismos de prestação de contas. De forma geral, as teorias relacionadas à NPM estão baseadas em uma racionalidade econômica e em relações claras entre objetivos, ações e resultados, associadas a sistemas de incentivos baseados em recompensas e punições.

Entretanto, o desenvolvimento das técnicas e instrumentos de gestão tem se mostrado insuficiente ante a crescente complexidade dos problemas enfrentados pelo Estado moderno; ou seja, eficiência é fator necessário mas insuficiente.

Em um mundo dicotômico, polarizado entre forças democráticas ocidentais (do bem) e regimes autoritários fechados (do mal), separados por uma "cortina de ferro», os problemas sociais tendem a ser simplificados, com respostas também dicotômicas e reducionistas. A pluralidade dos ambientes e contextos atuais implica em uma explosão de demandas e possibilidades de soluções, com crescimento exponencial da árvore de decisão.

Por sua vez, proliferam os problemas complexos e não solucionáveis pelas estruturas hierárquicas e arranjos funcionais. Problemas de saúde, com frequência, não podem ser solucionados pelas políticas do setor, mas dependem de melhorias na segurança pública (e redução das vítimas de violência e acidentes de trânsito), da educação (higiene e conscientização de medidas de prevenção) ou mesmo transporte (acesso às residências, tempo pessoal para medidas de higiene ou preventivas).

Estudo recente sobre os arranjos para execução do Programa Bolsa Família, programa federal brasileiro para transferência direta de rendas, que, segundo dados de fevereiro de 2016, atendia a 13,9 milhões de famílias, mostrou a necessidade de inúmeros mecanismos de coordenação intra e interinstitucionais. Ao utilizar diversas instâncias federativas - União, Estados e municípios - além de envolver repasses e controles entre estruturas funcionais distintas, das áreas de desenvolvimento social, saúde e educação, a execução dessa política exige mecanismos de coordenação sofisticados, não limitados à lógica vertical. Para um recurso da União alcançar o beneficiário final, é articulado um conjunto de atores, incentivos, controles e recursos.

O exemplo do Programa Bolsa Família visa evidenciar a crescente complexidade exigida na execução das políticas públicas, mostrando que a eficiência torna-se um problema menor ante as dificuldades de coordenação, e a centralidade da Administração Pública nessa execução não é adequada para sua efetividade. Essa complexidade pode se somar à amplitude das demandas, cujos reconhecimento e atenção se contrapõem ao hermetismo e inflexibilidade do Estado.

Este conjunto de novos problemas, denominado por Ferlie et al. (2011) como wicked problems $^{1}$, tem como característica não se limitarem a estruturas organizacionais ou áreas de política, envolvendo em sua solução ou direcionamento as atribuições de distintas organizações, públicas, privadas ou do terceiro setor. Para esses autores, os wicked problems se
O exemplo do Programa Bolsa Família visa evidenciar a crescente complexidade exigida na execução das políticas públicas, mostrando que a eficiência torna-se um problema menor ante as dificuldades de coordenação. 
caracterizam por exigirem o envolvimento de amplo conjunto de indivíduos e organizações, pela inexistência de uma solução óbvia ou direta; pela ausência de convergência ou concordância entre os atores envolvidos no problema ou em sua solução; e pela exigência de se incluir as mudanças de comportamento como parte da solução.

A proliferação dos wicked problems se contrapõe às modelagens mais tradicionais de políticas públicas, setorizadas ou hierárquicas. Nesse sentido, o tratamento dos wicked problems exige novos modelos de ação estatal baseados em redes, parcerias, coprodução, relações não hierárquicas, e, principalmente, a participação de atores não estatais no processo.

Novas soluções ou modelos para a ação estatal são então buscados a partir de uma mudança de natureza paradigmática, retirando do Estado a exclusividade na atenção às demandas da sociedade. $\mathrm{O}$ avanço dessas abordagens vem sendo tratado pela vertente da governança pública, que, como uma de suas principais características, considera um estado plural, no qual vários atores interdependentes contribuem para a prestação de serviços públicos, e também pluralista, em que vários processos e atores influenciam o sistema de tomada de decisões das políticas públicas (Osborne, 2006).

Se, na própria concepção, a NPM já implicava na transposição de conceitos e práticas entre setores (do privado para o público), sua migração e uso para outros países e culturas não deveria ter maior complexidade. O mesmo não ocorre, contudo, para a governança pública, muito mais imersa no ambiente politico, social e econômico, além do processo de construção histórica das relações de confiança entre os setores público, privado e terceiro setor.

Confiança, por sua vez, remete para a estabilidade das relações e das regras, e, em última instância, está profundamente associada às próprias instituições de um país. É nesse momento que aumentam os questionamentos quanto aos limites para a utilização da governança pública nos países da CPLP que, exceto por Portugal, encontram-se ainda em processo de construção ou solidificação de suas instituições, muitos recém se reerguendo de guerras e rupturas severas. É possível florescer um modelo baseado na confiança, nas regras tácitas, nesse contexto?

É útil concluir esta seção com a observação de Peci et al. (2008, p. 42), para quem:

«a governança deriva da cultura política do país onde se insere, enquanto a NPM não demonstra essa sensibilidade contextual e ideológica. Dessa forma, é de se esperar que os desenhos institucionais da governança sejam diferentes, dependendo do contexto onde são aplicados. De forma mais ampla, o conceito de governança pode ser utilizado na teoria de administração pública para qualificar as relações que o Estado (domínio dos políticos e burocratas) desenvolve com o setor privado (domínio das empresas e consumidores) e o terceiro setor (domínio da cidadania organizada em torno dos seus interesses).» 


\section{A Nova Governança Pública: redefinindo o papel do Estado?}

Apesar das críticas e, em muitos contextos, deficiência nas propostas, a NPM continua exercendo uma forte influência no pensamento administrativo, como evidenciado no Gráfico 1. Mas o gráfico evidencia, também, o crescimento no interesse pela governança pública principalmente nos últimos 15 anos. Essa seção apresenta uma breve evolução das ideias da governança e da NPM, como proposta por Osborne (2006), com o objetivo de destacar os principais aspectos exigidos para sua configuração, de modo a analisar as possibilidades e limitações de aplicação ao contexto dos países que integram a CPLP.

Para Osborne (2006), a gestão pública contempla cada vez mais uma articulação interorganizacional, envolvendo amplo conjunto de atores que participam não apenas na execução das políticas públicas mas também em sua identificação e concepção, o que remete à necessidade de uma visão sistêmica e processual em substituição a uma visão discreta e transacional. A Nova Governança Pública (NPG) muda a forma de articulação das relações institucionais entre setor público e sociedade, ampliando as redes entre organizações dentro e fora do governo para fornecer serviços públicos. A emergência de relações não hierárquicas inibe os sistemas de comandos formais, demandando a construção de relações baseadas na confiança e contratos relacionais não formais, segundo expectativas e possibilidades das partes.

O Estado torna-se, enfim, um organismo plural, exigindo mudanças nas teorias, ontologias e bases epistemológicas utilizadas para compreender tanto o papel do Estado nesse novo modelo quanto esse novo sistema de coordenação. Assim, as exigências de compreender o funcionamento das redes, os mecanismos de participação e representação dos grupos de interesse e colaboração na concepção, construção e produção de políticas públicas exige certo distanciamento das teorias econômicas, como da Public Choice e Agency Theory, e aproximação a modelos baseados na sociologia organizacional e teoria da rede.

Na realidade, as bases de um arcabouço de novas ideias em substituição ao referencial gerencialista da NPM já estavam apresentadas há mais de quatro décadas, com a proposta de Elinor Ostrom sobre as Public Service Organizations (PSO) e os modelos de coprodução, quando observou a partir das experiências do policiamento de bairro que a produção de serviços ao público tornava-se difícil sem uma ativa participação do cidadão que dele se beneficiaria. A coprodução, então, implicaria que os cidadãos assumissem um papel ativo na produção de bens e serviços públicos (Ostrom, 1996). A ideia das PSO, incorporando organizações públicas, privadas ou do terceiro setor, alavancou os modelos de parceria e coprodução, entendida como os relacionamentos regulares e de longo prazo entre provedores de serviço e seus usuários ou comunidade, e para a qual todas as partes fazem substancial contribuição de recursos (Osborne e Strokosch, 2013).

\section{As exigências}

de compreender o

funcionamento das redes, os mecanismos de

participação

e representação dos

grupos de interesse

e colaboração

na concepção, construção

e produção de políticas

públicas exige certo

distanciamento

das teorias econômicas, como da Public Choice

e Agency Theory, e aproximação a modelos baseados na sociologia organizacional e teoria da rede. 
Tanto na perspectiva da governança quanto da NPM ocorre a inclusão de agentes privados e do terceiro setor, mas as motivações e formas desse envolvimento são significativamente distintas. Na governança pública os vários atores participam do policy-making e do processo de implementação, sendo que o alinhamento social e a participação são considerados recursos fundamentais na materialização das políticas, inclusive com poderes para obstruir intervenções. Diferentemente, na NPM os agentes privados e da sociedade são incluídos diretamente no provimento dos serviços e políticas públicas, sem necessariamente participarem do diagnóstico do problema ou da concepção da solução. Ou seja, enquanto a governança busca integrar as partes - com as vantagens e dificuldades que isso acarreta - a NPM tende a produzir uma proliferação de atores e organizações distintas, cada qual especializada em sua própria tarefa (Klijn, 2012).

Mathiasen (2007) traz uma reflexão interessante sobre a difusão da NPM e as possibilidades de transferência de experiências em políticas públicas. Para o autor, o intenso debate, com argumentos favoráveis e contrários à NPM, somado à difusão internacional da NPM, permitiu construir as bases de um diálogo sobre o que é transferível e quais as condições sujeitas ao contexto político e cultural do país receptor, permitindo revisitar premissas implícitas na gestão pública que consideram o modelo da Europa atlântica como o mais desejável e objetivo das reformas no setor público.

Peters e Pierre (1998) analisam que, enquanto a NPM está relacionada a resultados, a governança trata mais dos processos. Ademais, em uma reflexão importante para o debate sobre as possibilidades de transposição das práticas, esses autores afirmam que a governança é um processo associado a um ambiente político e cultural, podendo se configurar de distintas formas segundo os contextos nacionais.

Rhodes (2007) caracteriza a governança pela interdependência entre as organizações (a governança é mais ampla do que o governo, incluindo intervenientes não estatais e alterando os limites do Estado), pelas interações contínuas entre os membros da rede; interações baseadas na confiança e reguladas por regras negociadas, e por um grau significativo de autonomia dos atores da rede em relação ao Estado. Entretanto, esse autor alerta para o problema do hollowing out of the state ${ }^{2}$, quando a capacidade do poder executivo central torna-se esvaziada por cima, pela interdependência internacional; por baixo, pelas redes; e pelos lados, pelas agências e organismos paraestatais, semelhante à metáfora do hollow state utilizada por Milward (1996) para descrever a crescente dependência do setor público em contratações de organizações sem fins lucrativos ou de firmas privadas para entregar os bens e serviços públicos.

Em realidade, há muitas propostas ou abordagens emergentes, surgindo como respostas às novas demandas e complexidades sociais. Para Bryson et al. (2014), apesar de não constituírem ainda um conjunto coerente, essa nova abordagem mostra convergência já em vários pontos, de que são 
exemplo as propostas de Janet e Robert Denhardt do chamado Serviço Público Novo, a gestão de valor público de Gerry Stoker, a publicização (publicness) de Barry Bozeman, a nova governança pública de Stephen Osborne (2010) e as novas políticas cívicas de Harry Boyte. Apesar das diferentes origens, as novas abordagens trazem em comum uma visão de um cidadão como parte da solução do problema e seu cocriador, de um governo como catalisador e colaborador mas por vezes direcionador, impulsionador ou simplesmente fora do caminho, e onde o público é visto como algo muito além do governo.

Não são poucos, contudo, os desafios para migrar de um modelo da NPM para a governança. O novo paradigma da NPM, da coprodução, e do valor público exige capacidade para lidar com uma desconcentração da ação pública, redução dos mecanismos hierárquicos de coordenação, distribuição de poder decisório a outros stakeholders (em geral, sem legitimidade política), fragilização dos sistemas estatais de controle e accountability ante inúmeros atores, atuando em torno das demandas públicas. Ademais, em uma estrutura de redes e parcerias deve ser resguardada aos agentes do Estado, inclusive ao sistema político, a capacidade de ouvir e representar de fato as expectativas da sociedade.

A difusão das ideias da governança pública pode, contudo, camuflar suas dificuldades, principalmente de transposição a outros contextos. De fato, inúmeros autores têm alertado para essas dificuldades, sendo Guerreiro Ramos um dos mais antigos, ao menos no contexto brasileiro, a levantar esse problema. Ao apresentar sua proposta de redução sociológica, a caracterizou como «um método destinado a habilitar o estudioso a praticar a transposição de conhecimentos e de experiências de uma perspectiva para outra. O que a inspira é a consciência sistemática de que existe uma perspectiva brasileira» (Ramos, 1996, p. 42).

Entre seus enunciados, propõe que a redução sociológica não admite a existência, na realidade social, de objetos sem pressupostos, uma vez que a realidade social é dotada de sentido, cujos significados são estabelecidos por suas conexões de sentido. Propõe ainda a redução sociológica como um procedimento crítico-assimilativo da experiência estrangeira, devendo essa assimilação ser adaptada ao local ou nacional, e os conceitos a serem transplantados reinterpretados pela cultura nacional para adequação ao seu conteúdo de sentido (Filgueiras, 2012).

Brown e Humphreys (1995) tratam da transferência de modelos ocidentais de administração para outras culturas. Com base em modelos de educação técnica do Reino Unido e seu uso no Egito, apontam que o potencial de transferência de modelos e práticas de gestão ocidental está repleto de dificuldades, repetindo as observações de Guerreiro Ramos quanto à necessidade de moldar e adaptar conceitos e práticas.

Daniell (2014) desenvolve uma extensa análise bibliográfica sobre o efeito dos padrões culturais nacionais sobre a configuração das políticas públicas.
As novas abordagens trazem em comum uma visão de um cidadão como parte da solução do problema e seu cocriador, de um governo como catalisador e colaborador mas por vezes direcionador, impulsionador ou simplesmente fora do caminho, e onde o público é visto como algo muito além do governo. 
Observa que as ideias políticas desenvolvidas em uma região são frequentemente transferidas a outros contextos, embora essa transferência pode não ser desejável ou implementável em virtude de inúmeros fatores de natureza sociopolítica, econômica, geográfica ou cultural que se mostram distintos.

Ademais, o comportamento dos governos e partes interessadas pode também variar consideravelmente entre países e regiões, sendo forte a relação entre características culturais, a forma e as razões associadas ao desenvolvimento das políticas públicas nos diferentes países. Nesse sentido, considera que há muito potencial para desenvolver a compreensão mais aprofundada das culturas nacionais e suas relações com a construção de políticas públicas dentro dos países, bem como sua transferência entre países.

Para Sen (2002), fatores culturais influenciam o comportamento econômico, a participação política, a solidariedade social e a associação, a formação dos valores e sua própria evolução. Para ele, a premissa econômica que todos os seres humanos se comportam da mesma forma é falha ante as evidências, uma vez que as influências culturais podem fazer uma grande diferença em questões relacionadas à ética, conduta responsável, empreendedorismo, disposição para assumir riscos, entre inúmeros outros aspectos fundamentais ao sucesso econômico de uma nação.

Esta breve revisão das propostas da Nova Governança Pública (NPG), inclusive das mudanças trazidas ante o ambiente anterior da NPM, permite antecipar desafios que precisam ser considerados para sua adequada incorporação como referencial da Administração Pública, ou mesmo para a possibilidade de adequação frente à ausência dos requisitos do contexto anglo-saxão que a suportam. É necessário então destacar esses requisitos, relacionados tanto a aspectos culturais quanto político e administrativos.

A definição aqui utilizada para cultura segue a proposta por Hofstede, ou seja, é a programação coletiva da mente humana que distingue um grupo ou categoria de pessoas do outro, ou aquilo o que muitos membros de um grupo têm em comum e pelo qual eles se distinguem de outros grupos, podendo ser identificados alguns componentes mais dinâmicos e outros relativamente estáveis. Assim, a cultura pode ser examinada, caracterizada e compreendida ao longo do tempo pelas relações entre pessoas e grupos, objetos, artefatos e sistemas organizativos.

Em consequência, uma cultura nacional está relacionada ao conjunto de crenças, comportamentos, normas e costumes compartilhados em uma nação, moldados por uma identidade social, contexto histórico, parâmetros econômicos, fatores institucionais e geografia (Daniell, 2014). Sua importância como elemento analítico e de grupamento de estudos é evidenciada no estudo de Minkov e Hofstede (2012), com base em dados de 299 regiões pertencentes a 28 países.

Nesse estudo, Hofstede identifica seis dimensões em que as culturas de diferentes países se diferenciam:

- Índice de distância do poder - indica como a sociedade lida com as 
desigualdades entre seus membros. Um baixo índice de distância do poder indica que as pessoas se esforçam para equalizar a distribuição do poder.

- Individualismo versus coletivismo - o coletivismo representa uma sociedade com preferência por laços culturais fortes, enquanto o individualismo exprime uma tendência a tomada de decisão de forma independente.

- Masculinidade versus Feminilidade - nas sociedades com alta masculinidade, as pessoas são movidas pela competição e pelos resultados em detrimento das relações de cooperação e dos valores relacionados a qualidade de vida.

- Aversão à Incerteza - como a sociedade tende a reagir diante de situações inesperadas. $\mathrm{O}$ alto índice de aversão a incerteza indica uma preferência para as situações controladas e previsíveis.

- Orientação para longo prazo versus curto prazo - as sociedades orientadas para o longo prazo tendem a respeitar as tradições e valorizar as relações perenes.

- Indulgência versus Restrição - culturas com alto índice de indulgência centram suas preocupações no presente e adotam um comportamento mais livre de regras sociais.

\section{Premissas dos novos paradigmas e exigências}

Dentre os desafios com que se defrontam os gestores públicos no esforço de transposição dos fundamentos da NPG em um contexto cultural distinto ao daquele de origem do modelo, os que se referem a convergência e a divergência dos valores, marcadores e traços culturais, tendem a ser os que apresentam maiores dificuldades de superação. O foco principal está na transmissibilidade de conhecimentos e práticas gerenciais entre culturas produtoras e receptoras (Motta, 2014).

O exame dos dados referentes aos índices de dimensões culturais dos países que integram a CPLP (Brasil, Portugal, Angola, Moçambique e Cabo Verde) e a dois dos países, Reino Unido e EUA, nos quais a NPG se origina, evidencia significativas diferenças (ver Quadro 2).

Se tomarmos, para efeito de análise comparativa, a média dos dois grupos - CPLP e RU-EUA, cujos dados estão dispostos no Gráfico 2 -, os grupos apresentam índices bastante distintos entre si, à exceção da dimensão que trata da indulgência, que não apresenta diferença significativa.

A diferenciação dos traços culturais nacionais entre os dois grupos pode significar uma barreira de difícil superação na transposição dos princípios do modelo da NPG ao ambiente cultural dos países da CPLP. A perspectiva da dependência da gestão em relação às variações nacionais deixa implícita a dificuldade de transferência de conhecimento (Mota, 2014).

$\mathrm{Na}$ medida em que as dimensões culturais nacionais condicionam a gestão, haverá um modo apropriado de adquirir e transferir os conhe- 
43|Revista de GESTÃO dos Países de Língua Portuguesa

Quadro 2 Índices de dimensões culturais por países

\begin{tabular}{|l|c|c|c|c|c|c|c|}
\hline & Brasil & Angola & Portugal & Moçambique & $\begin{array}{c}\text { Cabo } \\
\text { Verde }\end{array}$ & $\begin{array}{c}\text { Reino } \\
\text { Unido }\end{array}$ & EUA \\
\hline $\begin{array}{l}\text { Distância do } \\
\text { poder }\end{array}$ & 69 & 83 & 63 & 85 & 75 & 35 & 40 \\
\hline Individualismo & 38 & 18 & 27 & 15 & 20 & 89 & 91 \\
\hline Masculinidade & 49 & 20 & 31 & 38 & 15 & 66 & 62 \\
\hline $\begin{array}{l}\text { Aversão a } \\
\text { incerteza }\end{array}$ & 76 & 60 & 99 & 44 & 40 & 35 & 46 \\
\hline $\begin{array}{l}\text { Orientação de } \\
\text { longo prazo }\end{array}$ & 44 & 15 & 28 & 11 & 12 & 51 & 26 \\
\hline \begin{tabular}{l} 
Indulgência \\
\hline
\end{tabular} & 59 & 83 & 33 & 80 & 83 & 69 & 68 \\
\hline
\end{tabular}

Fonte: Elaborado a partir dos dados do Hofstede Center

cimento sobre o modelo? Ou ele se configura numa tentativa de imposição de práticas gerenciais de países desenvolvidos?

\section{Características comuns da Administração Pública nos países da CPLP}

A herança cultural e o idioma comum são os elementos que fundamentam a interação política e econômica e potencializam a mútua cooperação

Gráfico II Média das dimensões culturais países da CPLP versus RU-EUA

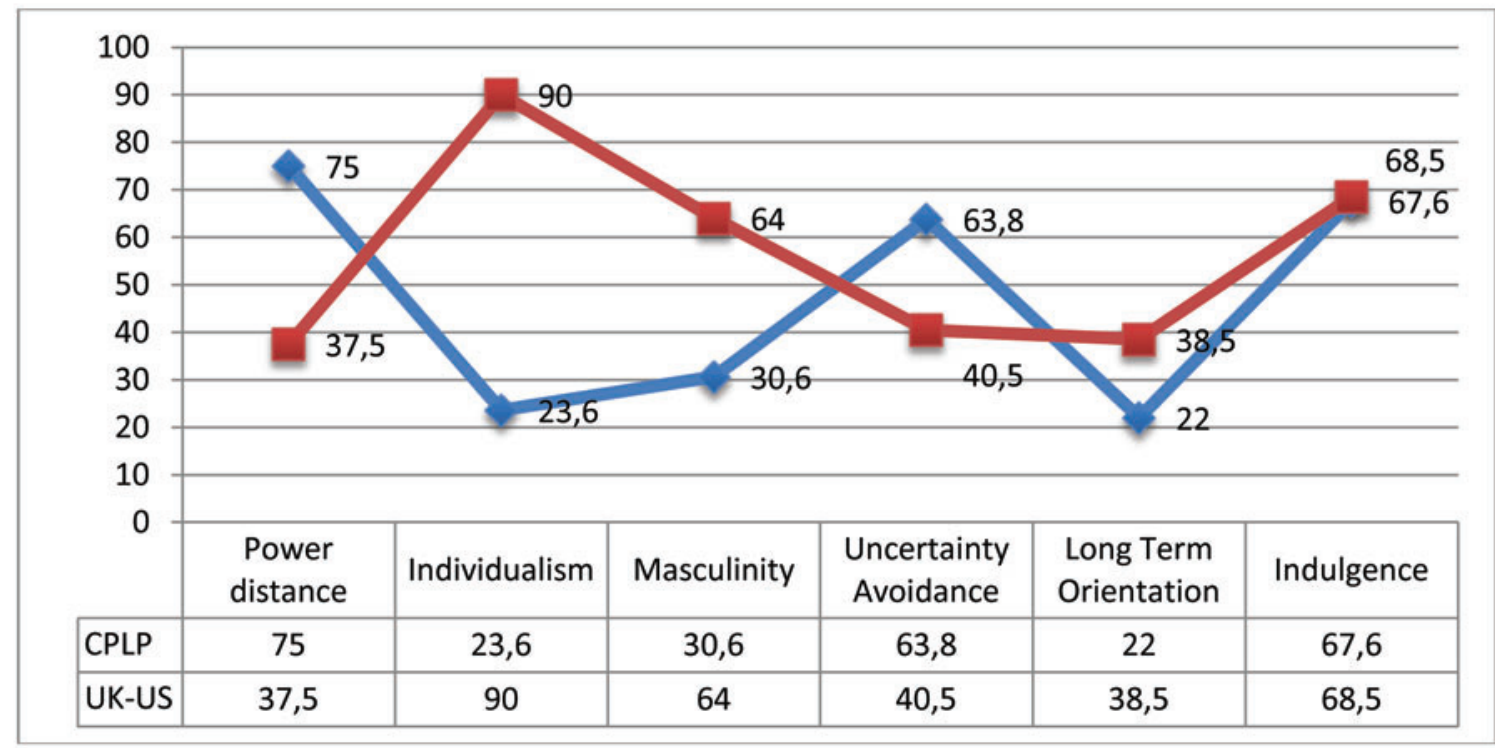

Fonte: Elaborado pelos autores

Obs: Azul relativo a CPLP; vermelho relativo a $R U$-EUA 
para o desenvolvimento na CPLP, atualmente composta por nove países situados em três diferentes continentes: Angola, Brasil, Cabo Verde, Guiné-Bissau, Guiné Equatorial, Moçambique, Portugal, São Tomé e Príncipe e Timor-Leste.

No domínio da Administração Pública, os esforços empreendidos no âmbito dessa cooperação têm se estabelecido, há quase duas décadas, em torno de temas prioritários relacionados a reforma e modernização administrativa, a formação profissional de recursos humanos ${ }^{3}$ e, mais recentemente, às questões referentes a adoção de sistemas de governo eletrônico ${ }^{4}$.

Paralelamente, as iniciativas de reformas administrativas conduzidas em cada um dos países nas últimas décadas seguem, em seus contornos mais amplos, as premissas do NPM, a despeito das especificidades inerentes aos momentos históricos, culturais, econômicos e políticos, próprios de cada nação.

Em Portugal, por exemplo, a reforma da Administração Pública assemelhou-se em muito àquelas presentes nos outros países ocidentais. $\mathrm{Na}$ prática, observou-se o estabelecimento de um clima de contradição, com a sobreposição e coexistência de modelos de administração com filosofias organizacionais e objetivos distintos: o modelo estatutário, o modelo político e o modelo gerencial, que privilegiava as propostas do NPM, ao mesmo tempo que preconizava a participação dos servidores públicos nos processos de mudança (Madureira, 2015).

No caso do Brasil, a ênfase da reforma do aparelho do Estado de 1995 centrou-se na gestão por resultados, com a implantação de planos estratégicos e o estabelecimento de metas de desempenho como fator de motivação dos recursos humanos. A reforma gerencial trouxe uma mudança de conceitos em toda estrutura administrativa do país, pois o objetivo era deixar o Estado mais gerencial e não patrimonial e burocrático (Bresser-Pereira, 2010).

Seguindo uma tendência mundial, a Administração Pública em Angola está sendo pautada em um conjunto de reformas no intuito de tornar seu Estado mais democrático e cidadão (Valadares et al., 2013). O modelo de Administração Pública Democrática Angolana é caracterizado por uma administração com uma superficial desconcentração e ainda centralizada. António (2004) avança que há a procura de uma gestão gerencial que tem como objetivo a melhoria do serviço prestado ao cidadão, o fortalecimento da carreira pública e a reforma do sistema de contratação de funcionários (Valadares et al., 2013).

O Estado moçambicano, por sua vez, promoveu uma reforma administrativa, levada a efeito em 2001, que teve como principais características a administração com enfoque na eficiência, transparência, accountability, e voltada para a prestação de serviços de qualidade aos cidadãos, segundo Simione (2014) (ver Quadro 3). A reforma iniciou uma tendência de criação de novas práticas voltadas à interação e participação públicas. 
O autor assevera acerca das barreiras à implantação dos princípios do NPM que as limitações estruturais e funcionais impostas pela adoção do modelo, em relação às metas de eficiência e obtenção de resultados e processos participativos, indica que os objetivos da criação de um novo

Quadro 3 Formas históricas do Estado e da Administração Pública em Moçambique

\begin{tabular}{|c|c|c|c|}
\hline Descrição & $1975-87$ & $1987-90-2001$ & $2001-11 \ldots$ \\
\hline $\begin{array}{l}\text { Natureza do } \\
\text { Estado }\end{array}$ & Estado Socialista & $\begin{array}{l}\text { Institucionalização do } \\
\text { Estado Capitalista e } \\
\text { criação do Estado } \\
\text { Democrático. }\end{array}$ & Estado Democrático \\
\hline $\begin{array}{l}\text { Orientação da } \\
\text { Administração } \\
\text { Pública }\end{array}$ & $\begin{array}{l}\text { Adoção de um } \\
\text { sistema de } \\
\text { administração } \\
\text { baseado nos } \\
\text { princípios da } \\
\text { burocracia } \\
\text { centralmente } \\
\text { controlada e } \\
\text { planejada. }\end{array}$ & $\begin{array}{l}\text { Coexistência entre } \\
\text { administração burocrática } \\
\text { do Estado e da burocracia } \\
\text { descentralizada no } \\
\text { contexto da criação das } \\
\text { autarquias locais. }\end{array}$ & $\begin{array}{l}\text { Adoção de novos } \\
\text { princípios de gestão } \\
\text { pública norteados pelos } \\
\text { padrões da Governança } \\
\text { Interativa (GI). }\end{array}$ \\
\hline $\begin{array}{l}\text { Características } \\
\text { da } \\
\text { Administração } \\
\text { Pública }\end{array}$ & $\begin{array}{l}\text { Exercício do controle } \\
\text { e autoridade das } \\
\text { estruturas } \\
\text { administrativas de } \\
\text { nível central e falta } \\
\text { de autonomia e de } \\
\text { poder de decisão dos } \\
\text { governos locais } \\
\text { (províncias e } \\
\text { distritos). }\end{array}$ & $\begin{array}{l}\text { Surgimento da } \\
\text { administração indireta do } \\
\text { Estado, privatização e } \\
\text { criação de formas } \\
\text { descentralizadas de } \\
\text { gestão pública e } \\
\text { estabelecimento } \\
\text { de estruturas } \\
\text { administrativas } \\
\text { autônomas. }\end{array}$ & $\begin{array}{l}\text { Transformações do } \\
\text { modelo de } \\
\text { administração } \\
\text { tradicional, } \\
\text { modernização e } \\
\text { institucionalização } \\
\text { primeiramente de } \\
\text { princípios da Nova } \\
\text { Gestão Pública (NGP) } \\
\text { e mais recentemente do } \\
\text { Novo Serviço Público } \\
\text { (NSP). }\end{array}$ \\
\hline $\begin{array}{l}\text { Escopo da } \\
\text { Administração } \\
\text { Pública }\end{array}$ & $\begin{array}{l}\text { Direção econômica, } \\
\text { técnica e } \\
\text { administrativa } \\
\text { vinculada às ações de } \\
\text { desenvolvimento } \\
\text { planificado do país. }\end{array}$ & $\begin{array}{l}\text { Prestação de serviços } \\
\text { públicos com novos } \\
\text { modelos de gestão que } \\
\text { promovem a eficiência } \\
\text { econômica do Estado e } \\
\text { autonomização dos } \\
\text { processos de tomada de } \\
\text { decisão. }\end{array}$ & $\begin{array}{l}\text { Administração com } \\
\text { enfoque na eficiência, } \\
\text { transparência, } \\
\text { accountability, voltada } \\
\text { para a prestação de } \\
\text { serviços de qualidade } \\
\text { aos cidadãos e } \\
\text { participativa na sua } \\
\text { gestão. }\end{array}$ \\
\hline
\end{tabular}

Fonte: Simione, 2014 
serviço público, modernizado e interativo, impõem que sejam estabelecidas ligações horizontais e verticais que ampliem e melhorem, de fato, a participação de novos atores na solução dos problemas das comunidades (Simione, 2014).

Reforça ainda que, apesar do esforço de existir grande interesse na aplicação de algumas práticas de articulação, ainda existem falhas na operacionalização dos mecanismos adotados, dado que as relações intergovernamentais são ainda fortemente condicionadas por problemas de natureza estrutural e organizacional e caracterizadas por grandes descontinuidades (Simione, 2014a).

Por conta da matriz cultural comum, permanecem latentes os traços culturais colonialistas que ainda estão presentes na Administração Pública da maioria dos países da CPLP, como o clientelismo, o corporativismo e o patrimonialismo.

\section{Desafios da NGP nos países de língua portuguesa}

Dado o framework das culturas nacionais, torna-se adequado investigar de que forma os requisitos da governança podem ser transplantados a novos contextos nacionais.

Assim, são elementos fundamentais a considerar na governança:

- O papel da sociedade civil organizada se torna fundamental, ao ampliar a capilaridade e flexibilidade da ação estatal via ações colaborativas;

- As relações de confiança entre Estado e sociedade devem permitir sustentar o desenvolvimento de projetos conjuntos e parcerias;

- A lógica da ação estatal deve migrar de uma base hierárquica para outra, baseada em redes e processos não hierárquicos;

- Um novo modelo mental para a atuação de servidores e políticos torna-se necessário, o que exige lidar com as resistências à mudança, corporativismo, estabilidade de carreira, sistemas de valores e recompensas;

- Para os políticos, novas formas de atuação, mais próximas às comunidades, tornam-se necessárias.

\section{Considerações Finais}

Nesse artigo, procuramos contribuir para a formulação de diretrizes para uma agenda de pesquisa que tenha como foco principal o incremento da produção de conhecimento inovador na temática da gestão das organizações na esfera pública dos países de língua portuguesa.

O intenso processo de transformações e mudanças recentes nos países da CPLP ressalta a importância do estudo de novas práticas de Administração Pública, com vistas a produção de inovações criativas, alinhadas ao processo de transformações da sociedade, que potencializem a modernização da gestão das organizações e tenham como principal finalidade a cooperação para o desenvolvimento.
Por conta da matriz cultural comum, permanecem latentes os traços culturais colonialistas que ainda estão presentes na Administração Pública da maioria dos países da CPLP, como o clientelismo, o corporativismo e o patrimonialismo. 
47| Revista de GESTÃO dos Países de Língua Portuguesa

\section{Notas}

1. Problema complicado, intrincado. Em sentido figurativo, problema cabeludo. N.E.

2. Esvaziamento do Estado. Um Estado que crescentemente usa terceiros para fornecer bens e serviços públicos e agir em nome do Estado. N. E.

3. Declaração do I Encontro de Ministros da Administração Pública da Comunidade dos Países de Língua Portuguesa, 1997.

4. 1. ${ }^{\text {a }}$ Conferência CPLP de Governo Eletrónico, 2013.

\section{Referências bibliográficas}

ANTÓNIO, P. R. (2004), «A reforma administrativa em Angola: Retrospectiva e perspectivas». In Congreso Internacional del CLAD sobre la Reforma del Estado y de la Administración Pública, IX, Madrid, 5 nov.

BRESSER-PEREIRA, L. C. (2010), «Democracia, crise e reforma». In M. A. D'Incao e H. Martins (Org.), Estudos sobre a Era Fernando Henrique Cardoso. Paz e Terra, São Paulo, pp. 171-212.

BROWN, A. D. e HUMPHREYS, M. (1995), «International cultural differences in public sector management». International Journal of Public Sector Management, vol. 8, issue 3, pp. 5-23 .

BRYSON, J. M.; CROSBY, B. C. e BLOOMBERG, L. (2014), «Public value governance: Moving beyond traditional Public Administration and the New Public Management». Public Administration Review, 74, 4, July, pp. 445-456.

DANIELL, K. A. (2014), «The role of national culture in shaping public policy: A review of the literature». HC Coombs Policy Forum, Crawford School of Public Policy, June, 2014. https://coombs-forum.crawford.anu.edu.au/publication/hc-coombs-policy-forum/4543/role-national-culture-shaping-public-policy-review. Acesso em 5/01/2016.

FILGUEIRAS, F. B. (2012), «Guerreiro Ramos, a redução sociológica e o imaginário pós-colonial». Caderno CRH, vol. 25, n. ${ }^{\circ}$ 65, pp. 347-363.

KLIJN, E. (2012), «New public management and governance: A comparison». Oxford Handbooks Online. http://www.oxfordhandbooks.com/view/10.1093/oxfordhb/9780199560530.001.0001/oxfordhb-9780199560530-e-14. Acesso em 14/03/2016.

MADUREIRA, C. (2015), «A reforma da Administração Pública central no Portugal democrático: Do período pós-revolucionário à intervenção da Troika». Revista de Administração Pública, vol. 49, n. ${ }^{\circ}$ 3, pp. 547-562.

MATHIASEN, D. (2009), «International Public Management». Oxford Handbooks Online. http://www.oxfordhandbooks.com/view/10.1093/oxfordhb/9780199226443.001.0001/oxfordhb-9780199226443-e-28. Acesso em 14/03/2016.

MILWARD, H. B. (1996), «Symposium on the hollow state: Capacity, control, and performance in interorganizational settings». Journal of Public Administration Research \& Theory, vol. 6, issue 2, Apr., pp. 193-195.

MINKOV, M. e HOFSTEDE, G. (2012), «Is national culture a meaningful concept? Cultural values delineate homogeneous national clusters of in-country regions». Cross-Cultural Research, 46(2), pp. 133-159.

MOTTA, P. R. M. (2013), «O estado da arte da gestão pública». Revista de Administração de Empresas, vol. 53, n. ${ }^{\circ}$ 1, jan/fev., pp. 082-090.

MOTTA, P. R. (2014), «Revisitando a controvérsia sobre a transmissibilidade intercultural do conhecimento administrativo». Cadernos EBAPE.BR, vol. 12, n. ${ }^{\circ}$ 3, p. 553.

MOTTA, P. R. e SCHMITT, V. G. H. (2013), «Administração para o desenvolvimento: A ideia e a renovação». Cadernos EBAPE.BR, vol. 11, n. ${ }^{\circ}$ 2, pp. 107-206.

OSBORNE, S.P. e STROKOSCH, K. (2013), «It takes two to tango? Understanding the coproduction of public services by integrating the services management and public administration perspectives». British Journal of Management, 24, S31-S47, Sept. 2. 
48 | Revista de GESTÃO dos Países de Língua Portuguesa

PECI, A.; PIERANTI, O. P. e RODRIGUES, S. (2008), «Governança e New Public Management: Convergências e contradições no contexto brasileiro». Organizações \& Sociedade, vol. 15, n. ${ }^{\circ}$ 46, julho/setembro, pp. 39-55.

PETERS, B. G. e PIERRE, J. (1998), «Governance without government? Rethinking public administration». Journal of Public Administration Research and Theory, vol. 8, no. 2, pp. 223 $-243$.

RAMOS, A. G. (1996), A Reducão Sociológica, Ed. UFRJ, Rio de Janeiro.

RHODES, A. W. (2007), «Understanding governance: Ten years on». Organization Studies, 28, pp. 1243-1264.

SEN, A. (2002), «How does culture matter?». In V. Rao e M. Walton (EE.), Culture and Public Action: Understanding the Role of Culture and Development Policy in an Unequal World, World Bank, pp. 37-58. http://tinyurl.com/ptg7u3q. Acesso em 20/08/2015.

SIMIONE, A. A. (2014), «A modernização da gestão e a governança no setor público em Moçambique». Revista de Administração Pública, vol. 48, n.o 3, pp. 551-570.

SIMIONE, A. A. (2014a), «Articulações intergovernamentais: Alcances e limites da coordenação e cooperação na gestão municipal em Moçambique». Cadernos Gestão Pública e Cidadania, vol. 19, n. ${ }^{\circ}$ 65, pp. 209-229.

VALADARES, J. L.; COSTA, K. G. D. S.; SILVA, E. A. e LUQUINI, R. A. (2013), «Brasil e Angola: Convergências e divergências epistemológicas sobre os modelos de Administração Pública». Revista de Ciências da Administração, vol. 15, n.o 36, pp. 131-142. 